\title{
Vom Referendum praecox zu Abortus ex utero und Phönix aus der Asche
}

Vom streitbaren Ständerat aus der Waldstatt wurden wir wegen unseres «Referendums praecox» abgekanzelt. Der rhetorische Missgriff wies in die richtige Richtung: Die mit praecox klinisch meist assoziierte genitale Funktionsstörung war symptomatisch für die gesamte KVG-II-Revision. Der Nationalrat hat der Bevölkerung erspart, dem so Gezeugten eine Abfuhr erteilen zu müssen. Der Abortus ex utero vom 17. Dezember 2003 war wohl die ziemlichste Lösung.

Die FMH hat sich zum richtigen Zeitpunkt als einzige Organisation klar gegen diese gesetzliche Missgeburt positioniert, die kein Problem gelöst, sondern nur neue geschaffen hätte. $\mathrm{Zu}$ einem Zeitpunkt, wo viele andere mit Wenn und Aber taktierten. Die klare Stellungnahme der FMH vom 11. Oktober 2003 hat entscheidend dazu beigetragen, diese unselige Vorlage zu bodigen, die nach Auffassung einer Vielzahl von Parlamentariern vor dem Volk nicht bestanden hätte. Verhindern eines unnötigen Referendums: ein substantieller Beitrag der Ärzteschaft zur Senkung der Kosten im Gesundheitswesen.

Ein schaler Nachgeschmack bleibt: Weshalb wollte man diese Gesetzesrevision mit hohler Schönrednerei am Volk vorbeischmuggeln? Die FMH als Präzeptor der schweizerischen Classe politique? Ob solchem mag man nicht recht glücklich werden. Unsere Sache ist, Patienten zu behandeln und Formen einer vernünftigen Gesundheitsversorgung zu entwickeln, nicht aber das (partei-)politische Tagesgeschäft. Wir wollen nicht ein weiteres Mal als die echten Fürsprecher des Volkes politische Selbstläufer zur Räson bringen müssen.

Wer allerdings in unseren Reihen glaubt, sich nun locker zurücklehnen und dem bisherigen Courant normal hingeben zu können, der un- terliegt einer fatalen Selbsttäuschung. Erfolg verpflichtet. Wir werden nun von allen Seiten durchaus zu Recht - aufgefordert werden, unsere Blueprints eines schweizerischen Gesundheitssystems vorzulegen, Antworten auf die drängenden Fragen wie Prämienwachstum, Effizienzsteigerung der Gesundheitsversorgung, Sicherheit der Behandlungen, Rationierung der Leistungen u. a.m. zu präsentieren. Für diese Antworten werden wir uns einmal mehr zusammenraufen müssen, weil in unseren Reihen zu etlichen dieser Fragen kontroverse Vorstellungen bestehen.

Massgeblich wird aber auch unser Tun sein, das Glaubwürdigkeit begründet. TARMED ist unser hic Rhodus, hic salta. Bei allen noch zu lösenden Problemen - Belegarzttarif, Verbesserung der Tarifstruktur für die operativen Disziplinen - haben die klugen und kompetenten Verhandlungen der kantonalen Gesellschaften und der FMH Rahmenbedingungen geschaffen, die für eine Einführung TARMED im ambulanten KVG-Bereich günstig sind. Die nun im ambulanten Spitalbereich entstandenen Probleme zeigen dagegen, was ungünstige Rahmenbedingungen und was Verhandlungen ohne Augenmass bedeuten können.

Die Öffentlichkeit wird in den kommenden Monaten sehr genau verfolgen, wie die FMH sich in gesundheitspolitischen Fragen positioniert und ob es ihr gelingt, TARMED in ihrem Einflussbreich einzuführen. Sollten wir wieder dem Schlendrian überheblichen Desinteresses verfallen, so wird der nun abortierte Fötus wie Phönix aus der Asche wieder erstehen. Ein fürchterlicher Phönix, dem dann auch das Kraut des Referendums nicht gewachsen wäre.

Hans Heinrich Brunner, Präsident FMH 